1056 LIVE SURGICAL BROADCAST AND PATIENT OUTCOME 10 YEARS OF EXPERIENCE FROM THE INTERNATIONAL CHARITÉ MAYO CONFERENCES 2010-2019

IJ Altmann*, ${ }^{1} \mathrm{R}$ Chekerov, ${ }^{2} \mathrm{C}$ Fotopoulou, ${ }^{3} \mathrm{~A}$ Du Bois, ${ }^{4} \mathrm{~W}$ Cliby, ${ }^{4} \mathrm{SC}$ Dowdy, ${ }^{4} \mathrm{~K}$ Podratz, ${ }^{1} \mathrm{~W}$ Lichtenegger, ${ }^{5} \mathrm{O}$ Camara, ${ }^{6} \mathrm{R}$ Tunn, ${ }^{7} \mathrm{D}$ Cibula, ${ }^{3} \mathrm{~S}$ Kümmel, ${ }^{8} \mathrm{~B}$ Vergote, ${ }^{9} \mathrm{~S}$ Chopra, ${ }^{9} \mathrm{M}$ Biebl, ${ }^{10} \mathrm{~V}$ Chiantera, ${ }^{11} \mathrm{~J}$ Neymeyer, ${ }^{1} \mathrm{Z}$ Muallem, ${ }^{1} \mathrm{JU}$ Blohmer, ${ }^{1} \mathrm{~J}$ Sehouli. ${ }^{1}$ Charité University Hospital, Gynecology, Berlin, Germany; ${ }^{2}$ Imperial College London, Gynecology, London, UK; ${ }^{3}$ Evangelische Kliniken Essen-Mitte, Gynecology, Essen, Germany; ${ }^{4}$ Mayo Clinic, Gynecology, Rochester, USA; ${ }^{5}$ Hufeland Kliniken Langensalza, Gynecology, Langensalza, Germany; ${ }^{6}$ Hedwig Kliniken Berlin, Berlin, Germany; ${ }^{7}$ General Hospital Prague, Prague, Czech Republic; ${ }^{8}$ UZ Leuven, Gynecology, Leuven, Belgium; ${ }^{9}$ Charité University Hospital, Surgery, Berlin, Germany; ${ }^{10}$ Gemelli Universita, Roma, Italy; ${ }^{11}$ Charité University Hospital, Urology, Berlin, Germany

10.1136/ijgc-2021-ESGO.327

Introduction/Background* The international Charité MAYO Conference aims at promoting international dialogue on diagnostics, management, scientific breakthroughs and state-of-theart surgical procedures in gynecology and gynecological oncology. Live surgeries are a fundamental tool of interdisciplinary and international exchange of experts in their respective fields. There is a controversy about the usefulness, risks and safety of live-surgical broadcasts. The aim of the current study is to analyse perioperative risks and oncological outcomes in patients who underwent live-surgery during the Charite MAYO Conferences.

Methodology Live-surgeries were performed by the core Charité team consisting of gynecologic oncologic surgeons, partly in collaboration with visiting gynecologic oncological surgeons. We performed a retrospective analysis of all live-surgeries performed during seven Charité MAYO Conferences from 2010 to 2019 held in Berlin, Germany.

Result(s)* In total, 69 patients underwent live-surgeries. 13 patients received urogynecological procedures, 21 patients underwent surgery on the breast and 35 patients underwent surgery due to gynecological cancer such as ovarian, uterine, vulvar or cervical cancer. Perioperative complications were classified according to the Clavien-Dindo classification. The rate of perioperative complications, macroscopic tumor resection and oncological outcomes were within the range published in the literature.

Conclusion* Based on our analysis live-surgeries were safe to perform in terms of multidisciplinary approach, perioperative complications and oncological outcome. With this, we established specific recommendations for performing livesurgeries.

\section{STANDARDIZING THE HIPEC PROCEDURE AND PERIOPERATIVE CARE FOR PATIENTS WITH OVARIAN CANCER IN THE NETHERLANDS USING A CONSENSUS- BASED DELPHI APPROACH}

${ }^{1} \mathrm{R}$ Van Stein*, ${ }^{1} \mathrm{C}$ Lok, ${ }^{2} \mathrm{~A}$ Aalbers, ${ }^{3} \mathrm{I}$ De Hingh, ${ }^{4} \mathrm{~A}$ Houwink, ${ }^{5} \mathrm{H}$ Stoevelaar, ${ }^{1} \mathrm{G}$ Sonke, ${ }^{1}$ WJ Van Driel. 'Netherlands Cancer Institute, Gynaecology, Amsterdam, Netherlands; ${ }^{2}$ Netherlands Cancer Institute, Surgery, Amsterdam, Netherlands; ${ }^{3}$ Catharina Hospital, Surgery, Eindhoven, Netherlands; "Netherlands Cancer Institute, Anesthesiologie, Amsterdam, Netherlands; ${ }^{5}$ Ismar, Lier, Belgium

\subsection{6/ijgc-2021-ESG0.328}

Introduction/Background* Based on the results of the randomized controlled OVHIPEC-1 trial, Hyperthermic Intraperitoneal Chemotherapy (HIPEC) with cisplatin has been incorporated in Dutch evidence based guidelines and is considered routine treatment in patients with stage III epithelial ovarian cancer following complete or optimal interval cytoreductive surgery (CRS). Differences in patient selection criteria, technical aspects and perioperative management exist between the ten Dutch centers that perform HIPEC for ovarian cancer. As part of an implementation process, we aim to standardize these important aspects using a consensus-based Delphi approach.

Methodology This consensus study comprised a two-phase modified Delphi approach involving 40 experts with experience in performing the HIPEC procedure for ovarian cancer (gynecological oncologists, medical oncologists, oncological surgeons and anesthesiologists). Key aspects of HIPEC were identified in panel discussions. Next, the expert panel completed a survey consisting of various statements and questions about these aspects. During a subsequent consensus meeting, survey outcomes were discussed considering the available scientific evidence. Consensus was defined as $\geq 75 \%$ agreement on a statement or question. Statements with $<75 \%$ agreement were adjusted based on feedback from the panel members. Level of agreement with the revised items is currently determined in a second survey.

Result(s)* In the first round, the response rate was 98\% and consensus was seen for $45 \%$ of statements and questions. Highest agreement was present for selected statements on CRS and postoperative care. For those statements consensus was reached, agreement varied from $78-100 \%$ (statements on CRS) and 78-95\% (statements on postoperative care). Items on patient selection, setting the indication, preoperative workup, and technical aspects of HIPEC required more panel discussion and revision. Results of the second round will be available by July 2021 .

Conclusion* A Dutch expert panel reached substantial agreement on a number of statements regarding HIPEC for ovarian cancer. Lack of consensus was generally associated with absence of robust scientific evidence. The results of this consensus study can help to align treatment protocols and minimize practice variation. These efforts will ensure optimal and safe introduction of HIPEC in the Netherlands, result in a safe treatment for all eligible patients and may guide the research agenda on HIPEC for ovarian cancer.

\section{INFLUENCE OF COVID-19 PANDEMIC ON STAGING OF OVARIAN CANCER - EXPERIENCE OF CERTIFIED ADVANCED OVARIAN CANCER SURGERY CENTER}

1J Dobroch* ${ }^{2}{ }^{2}$ Gronostajska, ${ }^{2}$ AK Strosznajder, ${ }^{2} \mathrm{~K}$ Kubica, ${ }^{2}$ Lenartowicz, ${ }^{2} \mathrm{G}$ Naronowicz, ${ }^{1} \mathrm{P}$ Knapp. ${ }^{1}$ Medical University of Białystok, Department of Gynecology and Gynecologic Oncology, University Oncology Center, Bialystok, Poland; ${ }^{2}$ Medical University of Białystok, Students' Scientific Group at the University Oncology Center, Białystok, Poland

\subsection{6/ijgc-2021-ESGO.329}

Introduction/Background* COVID-19 pandemic restrictions caused a limitation in healthcare services availability. This could lead to the delay in diagnosis and onset of the treatment. Ovarian cancer (OC) is frequently detected in advanced stage due to its asymptomatic development. The study aimed to determine whether the incidence and staging of OC were influenced by COVID-19 pandemic in the certified advanced ovarian cancer surgery center.

Methodology The study consisted of 77 patients with primary ovarian cancer admitted to the University Oncology Center in Białystok, Poland between march 2019 and march 2021. We 